University of Nebraska - Lincoln

DigitalCommons@University of Nebraska - Lincoln

Faculty Publications, Department of Psychology

Psychology, Department of

2020

\title{
Identifying and addressing barriers to treatment for child sexual abuse survivors and their non-offending caregivers
}

\author{
Kate Theimer \\ University of Nebraska - Lincoln, katetheimer4@gmail.com \\ Akemi E. Mii \\ University of Nebraska - Lincoln \\ Emily Sonnen \\ University of Nebraska - Lincoln \\ Kelsey McCoy \\ University of Nebraska - Lincoln \\ Katie Meidlinger \\ University of Nebraska
}

See next page for additional authors

Follow this and additional works at: https://digitalcommons.unl.edu/psychfacpub

Part of the Marriage and Family Therapy and Counseling Commons, Psychiatric and Mental Health Commons, Psychiatry Commons, and the Psychology Commons

Theimer, Kate; Mii, Akemi E.; Sonnen, Emily; McCoy, Kelsey; Meidlinger, Katie; Biles, Brittany; Huit, T. Zachary; Flood, Mary F.; and Hansen, David J., "Identifying and addressing barriers to treatment for child sexual abuse survivors and their non-offending caregivers" (2020). Faculty Publications, Department of Psychology. 995.

https://digitalcommons.unl.edu/psychfacpub/995

This Article is brought to you for free and open access by the Psychology, Department of at DigitalCommons@University of Nebraska - Lincoln. It has been accepted for inclusion in Faculty Publications, Department of Psychology by an authorized administrator of DigitalCommons@University of Nebraska - Lincoln. 


\section{Authors}

Kate Theimer, Akemi E. Mii, Emily Sonnen, Kelsey McCoy, Katie Meidlinger, Brittany Biles, T. Zachary Huit, Mary F. Flood, and David J. Hansen 


\title{
Identifying and addressing barriers to treatment for child sexual abuse survivors and their non-offending caregivers
}

\author{
Kate Theimer, Akemi E. Mii, Emily Sonnen, Kelsey McCoy, \\ Katie Meidlinger, Brittany Biles, T. Zachary Huit, \\ Mary Fran Flood, David J. Hansen \\ University of Nebraska-Lincoln, USA \\ Corresponding author - K. Theimer, Department of Psychology, University of Nebraska-Lincoln, \\ 238 Burnett Hall, Lincoln, NE 68588-0308, USA; email KateTheimer4@gmail.com
}

\begin{abstract}
Mental health treatment is a critical part of an effective and compassionate response to the disclosure of child sexual abuse (CSA). Given the vast negative consequences for children and families following CSA, engagement in treatment can benefit youth and their non-offending caregivers. Yet, these families face unique barriers to treatment initiation, adherence, and effectiveness. The identification of these barriers allows clinicians, researchers, and policy makers to increase treatment utilization, engagement, and value. The current review and its recommendations derive from the existing literature combined with knowledge gained from a clinical research team with more than 20 years of experience offering a treatment program for CSA survivors and their non-offending family members. The review organizes barriers around factors related to individual characteristics of children and caregivers, perceptions and beliefs commonly held following CSA, and challenging family interactions in the context of individual and group treatment for CSA. Finally, barriers related to systemic and societal factors are examined given the importance of
\end{abstract}

Published in Aggression and Violent Behavior 52 (2020) 101418

doi 10.1016/j.avb.2020.101418

Copyright (C) 2020 Elsevier Ltd. Used by permission.

Submitted 9 October 2019; accepted 3 April 2020; published 11 April 2020. 
understanding the legal and cultural context in which families seek and engage in treatment. Recommendations for further research, suggestions for clinicians, and considerations for policy change to decrease the identified treatment barriers for families impacted by CSA are provided.

Keywords: Child sexual abuse, Treatment, Barriers, Non-offending caregivers, Clinical practice

\section{Introduction}

Mental health treatment and supportive services for children who experience sexual abuse are necessary given the variety of well-documented negative consequences associated with victimization. Youth who experience sexual abuse are at an increased risk for developing symptoms related to depression, anxiety, and posttraumatic stress disorder (Klonsky \& Moyer, 2008; Paolucci, Genuis, \& Violato, 2001; Spataro, Mullen, Burgess, Wells, \& Moss, 2004). Child sexual abuse (CSA) has also been associated with conduct or behavioral problems, such as aggression, substance use, and risky sexual behavior (Frothingham et al., 2000; Maniglio, 2009). Following their child's disclosure, non-offending caregivers of survivors may also experience significant distress (Cyr et al., 2016); thus, treatment efforts often include these family members (van Toledo \& Seymour, 2013).

Effective, evidence-based interventions can provide families who experience CSA a way to cope with the negative effects of abuse (e.g., Cohen, Mannarino, \& Deblinger, 2017; Trask, Walsh, \& DiLillo, 2011). A variety of treatment models exist, including individual therapy for the youth, individual therapy for the non-offending caregiver, and conjoint sessions between the child and caregiver (Cohen et al., 2017; Trask et al., 2011). Parallel group therapy, in which a group for victims and a separate group for non-offending parents meet concurrently to discuss analogous topics, has also been effective (e.g., Hubel et al., 2014; Stauffer \& Deblinger, 1996). Research suggests that a caregiver's involvement in treatment following their child's disclosure is linked to positive outcomes for the caregiver as well as the child (Deblinger, Stauffer, \& Steer, 2001; Domhardt, Munzer, Fegert, \& Goldbeck, 2015; Elliott \& Carnes, 2001).

In clinical practice, CSA may be broadly defined as any inappropriate interaction of a sexual nature between an adult and a youth (Haugaard, 2000). Further, CSA can include inappropriate sexual 
interactions between minors, when one minor is exerting power over the other (World Health Organization, 1999). Sexual abuse encompasses acts where the offender makes physical contact with the victim (e.g., fondling; digital, oral, vaginal, or anal penetration) as well as acts where the offender does not make physical contact (e.g., exposure to pornography). Although survivors of CSA are a diverse group whose sexually abusive experiences vary, treatment can help reduce the negative consequences experienced by youth and their non-offending family members (Kendall-Tackett, Williams, \& Finkelhor, 1993; Yancey \& Hansen, 2010).

While there are many challenges to treatment for children and families seeking mental health services in general (e.g., cost of therapy, lack of transportation, see Mojtabai et al., 2011 for a review), CSA survivors and their families may face additional unique barriers to treatment. Thus, victims and their families may experience the typical barriers to mental health care plus distinct barriers related to sexual abuse - compounding the number of challenges faced by these families.

These barriers may inhibit families from seeking and initiating therapy by reducing the likelihood a family seeks therapeutic service options, accepts a therapy referral, calls the therapy provider, and attends the first session. Barriers may reduce treatment adherence, including treatment attendance, participation and engagement in sessions, and completing homework or practicing skills outside of session. Interrelated with the effects on treatment initiation and adherence, these barriers may limit treatment effectiveness. Effective treatment may include increasing healthy coping skills and reducing the negative effects associated with the abuse (Cohen et al., 2017; Trask et al., 2011; Yancey \& Hansen, 2010). Thus, identifying and addressing these challenges are critical for best supporting children and families following CSA.

\subsection{Purpose}

The purpose of this paper is to describe the potential barriers specific to treatment for child sexual abuse survivors and their non-offending caregivers and to examine the challenges mental health practitioners may face when providing services. Suggestions for overcoming identified barriers and increasing treatment initiation, adherence, 
and effectiveness are provided for clinicians, researchers, and policy makers. The challenges described for individual, caregiver, family, and parallel group therapy for CSA are derived from the existing literature combined with practical knowledge gained from more than 20 years of clinical experiences offering a research and treatment program for CSA survivors and their non-offending family members (Hubel et al., 2014; Sawyer \& Hansen, 2014; Tavkar \& Hansen, 2011). Non-offending caregivers are a significant aspect of discussion throughout the paper as parents play a key role in their child initiating and completing therapy (Yasinski et al., 2018). Caregivers are responsible for accepting mental health referrals for their family or looking into services and contacting providers to set up therapy. Plus, caregivers must consent to treatment, and, in many cases, drive the child to services. Parents often communicate with the child's provider throughout the therapeutic process to provide valuable information aiding conceptualization and treatment goals. As noted, caregivers may receive their own services as well due to distress caused by the sexual abuse (van Toledo \& Seymour, 2013) and play an important role in their child successfully coping with CSA.

The paper begins by describing individual characteristics of children and caregivers that may act as barriers to treatment. The perceptions and beliefs of caregivers and children subsequent to CSA and the challenging interactions family members may face are then discussed as they relate to barriers to therapeutic intervention. Next, the unique difficulties experienced in group treatment are examined. Finally, while the context of the family system is considered for all barriers explored, wide-reaching systemic and societal factors are described as they introduce challenges for treatment. Each section is organized in a loose chronological order, with barriers relating to treatment initiation at the beginning followed by barriers relating to treatment adherence and effectiveness. However, many barriers associate with all three components - initiation, adherence, and effectiveness. Table 1 summarizes the identified barriers. Given the dearth of literature on barriers to treatment for CSA survivors and their family members, the paper concludes with recommendations for clinical practice, research, and policy.

Of note, this paper applies to situations where the child has disclosed or the abuse has become known through other sources. With numerous reasons for non-disclosure, many youth wait weeks, months, 
Table 1 Barriers to treatment initiation (I) and adherence (A) for child sexual abuse survivors and their non-offending caregivers.

Child and caregiver individual characteristics

- Hesitance to discuss abuse and emotions with a stranger

- Avoidance of uncomfortable topics (e.g., trauma, sex ed.)

- Caregiver requires unwilling/uninterested child to attend therapy

- Child's continued contact with the perpetrator (e.g., school, peer groups) $\quad \checkmark \checkmark \checkmark$

- Caregiver's own history of sexual abuse and subsequent therapy experiences $\checkmark \checkmark \checkmark$

- Caregiver prioritizes own emotional distress during therapy

- Caregiver's knowledge of age-appropriate sexual development and behavior $\checkmark$

- Caregiver's values and beliefs regarding sex education

Child and caregiver perceptions and beliefs

- Caregiver does not fully believe child's narrative of CSA experience

- Child or caregiver does not view the experience as sexual abuse

- Negative views of therapy or poor previous experiences with therapy

- Child has experienced prior CSA and did not attend treatment

- Confusion about interpreting and responding to child behaviors and emotions

- Negative expectations about the child's future functioning

- Blame attributed to child by caregiver

- Child and caregiver high levels of self-blame and guilt

- Cultural experiences which shape negative beliefs and perceptions

Family interaction

- Secondary trauma and family upheaval which reduces support for child $\quad \checkmark \checkmark \checkmark \checkmark$

- Family's continued relationship with perpetrator (e.g., family member) $\quad \checkmark \quad \checkmark$

- Ending relationship with perpetrator causes financial trouble or lack of support $\checkmark \quad \checkmark$

- Extended family members do not believe child and withdraw support $\quad \checkmark \checkmark \checkmark \checkmark$

- Prioritizing family cohesion over individuals' needs

- Tension between co-parents on decisions or reactions related to child

- Family members' incongruent coping styles

- Non-biological parents who enter child's life after CSA may feel disengaged

- Difficulty securing childcare for siblings

Group interaction

- Children and parents are concerned about group confidentiality and privacy $\checkmark \checkmark \checkmark$

- Group members worry their CSA experience will be too different from others $\checkmark \checkmark$

- Group members have strong negative reactions to hearing others' experiences $\quad \checkmark$

- Expression of mixed feelings about offenders discourages open sharing $\checkmark \checkmark$

- High level of parental self-focus affects group dynamic $\checkmark \checkmark$

- Accommodating individual needs and values disrupts group dynamic $\checkmark$ Systemic and societal factors

- Anxiety, self-blame, or uncertainties regarding court verdicts and outcomes $\quad \checkmark$

- Negative experiences, fears, distrust, or frustration with the legal system $\quad \checkmark \quad \checkmark \quad \checkmark$

- Racial and cultural biases when assigning blame to victim $\quad \checkmark \checkmark \checkmark$

- Legal issues specific to the abuse which influence child custody cases $\quad \checkmark \checkmark \checkmark$

- Social and cultural stigma and blame surrounding CSA $\quad \checkmark \checkmark \checkmark$

- Gender norms and scripts (e.g., additional stigma for male victims) $\quad \checkmark \quad \checkmark$

- Stigma surrounding victims who identify as LGBTQ+ $\quad \checkmark \checkmark \checkmark$

- Confusion and exhaustion for families working with numerous professionals $\checkmark \quad \checkmark$

Note. If a barrier associates with initiation (I) and/or adherence (A), then treatment effectiveness will also be impacted. 
or years to share their story and some never come forward (London, Bruck, Ceci, \& Shuman, 2005). Thus, a child's lack of disclosure or delay in disclosure can completely inhibit or postpone treatment specific to CSA. Among the host of barriers to disclosure, younger children, males, youth from certain family conditions (e.g., families who do not discuss issues related to sex, families with patriarchal attitudes or rigid gender roles), and those in cultural groups with high levels of shame or stigma surrounding CSA may be less likely to disclose, which directly influences access to care (Alaggia, Collin-Vezina, \& Lateef, 2019; Brazelton, 2015; Fontes \& Plummer, 2010).

\subsection{Child and caregiver individual characteristics}

As one of the three pillars of evidence-based practice, client characteristics and values are an essential consideration in treatment (Sackett, Rosenberg, Gray, Haynes, \& Richardson, 1996), particularly within the provision of family services following a youth's disclosure of CSA. A range of both child and caregiver individual characteristics may inhibit families from seeking and adhering to therapy. Further, as suggested by the bioecological model (Bronfenbrenner \& Morris, 2006), the interactions among individual family members influence child development and family functioning (e.g., Miller \& Dwyer, 1997; see section on family interaction barriers). Thus, the interaction between varying child and caregiver characteristics may play a role in a family's therapeutic engagement.

Following the disclosure of sexual abuse a child may be apprehensive about seeking mental health services for many different reasons. Youth may fear disclosing personal or sensitive information to a stranger (Gulliver, Griffiths, \& Christensen, 2010) or have hesitation about discussing their thoughts and feelings related to the CSA with someone that they do not know, which may prevent initiation of treatment (Gopalan et al., 2010). Research suggests that youth, particularly those in distress, may actively seek to disengage from traumarelated content (Asmundson, Stapleton, \& Taylor, 2004), which then could contribute to premature treatment termination. Alternatively, youth may experience discomfort related to specific topics in treatment (e.g., sex education) that may lead to avoidance or disengagement in sessions where these topics are discussed. If a child is not willing to attend therapy, their caregiver may require them to attend 
involuntarily, impacting their engagement throughout the course of treatment. Given the focus of CSA treatment, cultural and religious beliefs can contribute to apprehension about seeking or engaging in therapy for some youth and their families (Fraynt et al., 2014). Notably, in-session avoidance by both the child and caregiver have been found to predict child outcomes (Yasinski et al., 2016) and drop-out (Yasinski et al., 2018) in trauma-focused therapy, which suggests that avoidance may play an especially important role in treatment adherence following CSA.

A youth's ongoing contact or relationship with the perpetrator may be a barrier to seeking therapy and can negatively affect treatment engagement. Youth who are victims of peer abuse may have a continued relationship with their perpetrator in the context of school or peer groups potentially leading to difficultly adhering to treatment. For example, a common component to CSA treatment includes discussion about the offender (Tavkar \& Hansen, 2011), which may be challenging for a child who is still interacting and communicating with their perpetrator. As discussed in detail later (see section on family interaction), the relationship between the perpetrator and the family (e.g., the perpetrator and caregiver have an ongoing relationship) may negatively impact parent and child interactions as well as both individuals' adherence to treatment (Bolen \& Lamb, 2004).

A caregiver's own history of sexual abuse may influence the family's involvement in treatment (Elliott \& Carnes, 2001). Caregivers who experienced sexual abuse as a child may show greater motivation to seek mental health services for their family and adhere to treatment because their own experience demonstrated the importance of therapeutic support for a child following abuse (i.e., the caregiver received therapy that was beneficial or they experienced distress because they did not receive support). However, a caregiver's history of negative experiences with therapy may diminish their desire or ability to seek and adhere to treatment, especially if they believe that it would be unhelpful or harmful (Cohen \& Mannarino, 1996). Research indicates that caregivers' expectations for therapy and perceptions of treatment relevance predict dropout from therapy (de Haan, Boon, de Jong, Hoeve, \& Vermeiren, 2013; Kazdin, Holland, \& Crowley, 1997). Further, a child's perception of their caregiver's approval of treatment has been shown to predict retention (Ormhaug \& Jensen, 2018). 
Caregivers' CSA history may contribute to emotional issues, separate from their child's abuse, that surface during a child's treatment. Such concerns may lead a caregiver to be avoidant, disengaged, or focused on processing emotions related to their own abuse instead of their child's. If a caregiver exhibits a high-level of self-focus in therapy, whether related to their own experience of sexual abuse or solely about how their child's sexual abuse has impacted them, then one of the primary goals of therapy (providing support to the child) may be hindered and treatment efficacy may be impaired.

Caregiver knowledge regarding age-appropriate sexual development and behavior may also limit effective treatment (Miller \& Dwyer, 1997). When caregivers do not have basic knowledge of age-appropriate sexual development they may not recognize when their child is exhibiting inappropriate behaviors, thus, allowing the inappropriate behavior to continue without remediation. Alternatively, a caregiver may pathologize their child's developmentally appropriate behavior and attribute it to the sexual abuse. Parents may also provide inaccurate information to their children regarding development and sexual behavior as a reflection of their unique personal or family values.

Sex education is a common component of CSA treatment given the importance of children understanding their own bodies and their agency following an unsafe sexual experience (Cohen et al., 2017; Hubel et al., 2014). However, caregivers present to treatment with varying levels of acceptance of sex education, particularly when taught to young children. Religious and cultural beliefs often influence comfort with sex education, and caregivers may feel it is inappropriate or inconsistent with their values for their child to learn about sexual development. Sometimes caregivers choose to skip sessions related to this material or withdraw from treatment altogether.

\subsection{Child and caregiver perceptions and beliefs}

Child and parent perceptions, attitudes, and beliefs following CSA can become barriers to initiating and engaging in treatment. Literature shows that non-offending parents' negative reactions or disbelief following their child's disclosure of CSA can predict future psychological problems for children, and those problems may continue into adulthood (Roesler \& McKenzie, 1994; Ullman, 2002). Similarly, victims' maladaptive interpretations of the abuse (e.g., blaming oneself for the 
abuse) and the psychological health effects these interpretations may have can act as treatment barriers (Zinzow, Seth, Jackson, Niehaus, \& Fitzgerald, 2010). Importantly, factors related to a client's gender identity, sexual orientation, background, culture, and individual experiences shape these perceptions and beliefs, as discussed in later sections of this paper (also see Fontes \& Plummer, 2010 for a review).

Parents are unlikely to seek mental health treatment for their family if they do not believe their child's disclosure (Bolen \& Lamb, 2004). Similarly, parents may be less likely to seek treatment if they believe what their child says, but do not believe it constitutes sexual abuse (e.g., child was exposed to pornography or fondled over the clothes, but parent does not view such behaviors as abusive). Likewise, some children may not see their experience as abusive. Although believing they were not negatively impacted by the experience can be protective, such beliefs may also keep children from the benefits of treatment, particularly if denying the assault or its effects is an effort to avoid discussing their feelings (Domhardt et al., 2015). This can be especially true for children who were extensively groomed prior to the assault, as the lines between the offender's "special attention" and sexual assault may become increasingly blurred (Craven, Brown, \& Gilchrist, 2007).

Some parents may become involved in their child's treatment even if they do not fully believe their child's description of the event or perceive it as abusive. A common example of this is when one parent believes the child, while the other parent is unsure or doubtful but feels pressured to attend therapy. In this case, treatment engagement will likely be impaired for the parent who does not believe the child. In turn, when this disbelief is overtly or unintentionally conveyed to the youth, the child may question the validity of their own CSA experience or their worthiness for help, causing difficulties for processing their emotions in treatment (Malloy, Mugno, Rivard, Lyon, \& Quas, 2016).

Even when a parent believes the disclosure and both the parent and child view the event as sexually abusive, there are additional perceptions of the abuse that can become barriers to treatment initiation and engagement. For example, there may be specific barriers for families who sought treatment for a prior CSA incident and later experience a second CSA incident. If parents or children had negative or unhelpful past experiences with therapy for CSA, this can cause frustration and distrust in mental health services, leading to difficulty initiating 
or engaging in treatment for families with children who have been revictimized (Kerkorian, Bannon Jr, \& McKay, 2006). Conversely, if a child was victimized in the past and did not receive therapy at that time, families may fail to see a reason to attend therapy following a second CSA incident.

Another common difficulty for parents of CSA victims is general confusion about how to respond to children's behavioral or emotional problems. Many non-offending parents feel guilty about their child's experience (Hill, 2001) and may act permissively toward problematic child behaviors, such as allowing the child to skip therapy sessions. Some parents may believe that their child's emotional problems are an unchangeable result of experiencing sexual abuse (Holguin \& Hansen, 2003) and therefore fail to model or communicate the importance of treatment engagement. Relatedly, parents and others may feel that there is no hope for survivors of CSA - that they are "damaged goods" or "forever changed" (Holguin \& Hansen, 2003; Theimer \& Hansen, 2018). This misconception can arise from the stigma that surrounds the label of the "sexually abused child" (Holguin \& Hansen, 2003; see section of this paper on systemic and societal factors). However, parents' confidence in their child's ability to cope, return to normal functioning, and be successful later in life are important for recovery (Kouyoumdjian, Perry, \& Hansen, 2009). When a parent has negative expectations for their child's future functioning, there can be detrimental effects that extend beyond the impact of the abuse itself (Kouyoumdjian et al., 2009). Morrissey-Kane and Prinz (1999) found that when parents had more negative cognitions and attributions related to their children's behavior problems, they were less likely to seek professional help, less likely to be engaged during treatment, and more likely to drop out of treatment. Additionally, children may be affected by the label of sexual abuse victim (Holguin \& Hansen, 2003) and have difficulty believing that therapy will help them.

The societal belief of CSA victims as blameworthy (see systemic and societal factors; Feiring, Taska, \& Chen, 2002; Ullman, 2002) can influence parent and child perceptions. Such social responses can be especially salient in situations where the youth engaged in risky behaviors (e.g., using alcohol or drugs, conversing with strangers online) and are subsequently assaulted. Parents may have difficulty seeing past the rule-breaking behaviors and end up assigning blame to the child for what happened. When this occurs, parents may struggle 
to support their child in therapy or have difficulty engaging in treatment themselves because they are overly focused on what their child could have done to prevent the assault. If a child perceives that their parent blames them for the assault, they may internalize these feelings (Hunter Jr, Goodwin, \& Wilson, 1993), leading to reduced treatment adherence.

Even without experiencing external blame, a common response to sexual abuse for both youth and non-offending parents is the feeling of self-blame and guilt (Feiring et al., 2002). When children try to understand why the abuse has happened to them, they may believe it happened as a result of their own mistakes (e.g., thinking they should have known the offender's intentions, disclosed earlier, or fought back against the perpetrator; Feiring \& Cleland, 2007). Youth may also blame themselves for the family turmoil which commonly follows abuse disclosures (e.g., divorce, financial stress, emotional distress in parents and siblings). Likewise, parents may perceive that they failed to protect their child, feel guilt over choosing an abusive partner or care provider (e.g., coach, teacher, priest, health care), or doubt their parenting abilities following the disclosure (Runyon, Spandorfer, \& Schroeder, 2014). It is likely that children and parents may be disengaged in therapy, minimize their symptoms to avoid further distressing others, or fail to retain skills taught in treatment because of perseveration on self-blame for the abuse and its effects on the family.

\subsection{Family interaction}

The bioecological model indicates that characteristics, perceptions, and beliefs of individual members of a family interact within the family system (Bronfenbrenner \& Morris, 2006) and are an important consideration when providing treatment. Outcomes for victims are closely related to the level of support the youth receives from other members of the family (Domhardt et al., 2015; Runtz \& Schallow, 1997). However, support may be impeded by family members' experiences of secondary trauma and their reactions to family upheaval subsequent to allegations of CSA (Cohen \& Mannarino, 1998; Elliott \& Carnes, 2001).

Beyond psychological difficulties after learning of CSA, logistical and structural changes can introduce transitional stressors within a family (Wolfe \& Jaffe, 1991). Offenders tend to be people close to the 
family or members of the family (Craun \& Theriot, 2009), which complicates how victims and non-offending family members navigate their relationships with the perpetrator. While contact between the victim and perpetrator is often limited or severed through legal efforts or the volition of the family, sometimes family members do not or cannot fully terminate a relationship with the offender. For example, the non-offending parent sometimes continues to engage in a relationship with the perpetrator as a significant other and may struggle to prioritize the well-being of the family as a whole, which may prevent treatment initiation and adherence. Ongoing relationships with the perpetrator allow the perpetrator to have continued opportunities to manipulate perceptions and choices made within the family system, including decisions surrounding if and how the family approaches treatment. Some culturally based family traditions emphasize the importance of family cohesion, which may make separation from the perpetrator an unacceptable option for the family. Cultures that promote stark power differentials between family members (Alaggia et al., 2019; Futa, Hsu, \& Hansen, 2001) may impede the non-offending caregiver's ability to make an autonomous decision about the status of the relationship with the perpetrator.

Still, termination of relationships between the perpetrator and all family members frequently introduces a variety of barriers to treatment as well. If the perpetrator was a significant other or caregiver, sudden changes in the family's financial situation, childcare needs, or family system dynamics can be major obstacles to treatment initiation or adherence (Plummer \& Eastin, 2007). The non-offending parent may struggle to adapt to their new role as a single parent or find it necessary to take on multiple jobs to cover the family's expenses. Treatment is often relegated to a lesser priority in families with unstable finances, as basic needs become harder to cover (Gopalan et al., 2010).

In cases where neither parent is the offender, and financial circumstances and family make-up remain stable, other barriers may affect treatment engagement. Co-parents often differ in their reactions to learning about the sexual abuse as well as their perspectives and approaches to treatment. These differences can contribute to varied responses and desires to initially engage in and adhere to treatment. In some cases, one parent may express disbelief or denial that the sexual abuse occurred or misplace blame to the victim, while the other 
parent expresses belief and support. This discrepancy can create tension between family members and influence whether a family initiates and engages in treatment, with one caregiver potentially not receiving treatment at all. In families with two supportive caregivers who are able and willing to attend therapy, the disclosure of abuse can alter parenting and coping styles adding disruption as new rules and response patterns are introduced to the family system. For example, many parents react to abuse by tightening their monitoring in a way that restricts their child's social interactions (Banyard, Rozelle, \& Englund, 2001). Other parents may attempt to overcompensate for the child's abuse experience such that they become less likely to enforce rules or expectations. Sudden changes in family dynamics and parenting practices can act as barriers to treatment.

Many step- and foster-parents who joined the family after the abuse occurred have pursued and engaged in treatment with some evidence that foster families have lower rates of treatment dropout for various reasons (Yasinski et al., 2018). However, caregivers who assumed a parental role after the abuse occurred may experience feelings of disconnection from the abuse (Yasinski et al., 2018). This can lead to less engagement in the treatment process and may be viewed by others as inattention or lack of concern.

Additionally, the presence of non-victimized siblings can act as a barrier to services for families. The additional burdens of finding and paying for reliable childcare for siblings who are not participating in treatment may prevent the family from engaging in therapy altogether. Some families choose not to disclose the abuse to the other children in the family, which can create additional tension surrounding the logistical complications of attending treatment. Whereas, nonabused siblings who know about the abuse may differ in their support of their sibling depending on how much they believe the disclosure. Sibling knowledge of the abuse allegation and sibling support can influence the caregiver's ability to participate in services. For example, responding to the needs of a sibling who was not abused is often an additional stressor for caregivers and survivors who may otherwise fully engage in treatment.

Furthermore, extended family dynamics can impact caregivers' and children's willingness to initiate and engage in treatment. This is a particularly relevant experience for families who face non-support and non-belief from extended family members, and they may receive 
pushback or criticism for engaging in treatment. Such dynamics become especially pronounced when the offender is a part of the family system, which can create divisions in families and provide additional barriers to treatment initiation, adherence, and effectiveness. For example, in cases where the unsafe touch is from another child in the family, a parent who faces balancing the needs of a child who engaged in problematic sexual behaviors with the needs of a child who is a victim may not be able to adequately engage in treatment. Additionally, familial dynamics may be impacted substantially as extended family have differing opinions on acknowledging the sibling's problematic sexual behaviors as well as the decision to seek treatment for either the victim, the sibling, or both. The influence of the extended family may also vary based on cultural context, and some families may experience barriers based on who can make decisions and how decisions are made within the family (e.g., significance of elders and their ability to make decisions on behalf of the family; Futa et al., 2001).

\subsection{Group interaction}

Group treatment for CSA offers valuable opportunities for youth and their families to receive emotional support and insights from others who have similar experiences. It also has efficiencies that make it more financially feasible for families who have resource limitations or who find it difficult to afford treatment for multiple family members. Disparities in access to treatment exist for racial and ethnic minority groups, including the ability to pay for services for multiple family members (Atdjian \& Vega, 2005). Therefore, group interventions expand treatment availability and may be more cost effective as multiple families are able to simultaneously receive services. However, in addition to the benefits of group interventions, unique barriers can affect participation.

As mentioned in prior sections, children and adolescents may be concerned about confidentiality and, therefore, be less likely to initiate group treatment. Within the group setting, youth and caregivers may be preemptively concerned about the possibility of knowing someone in the group or that other group members will not keep their information private, including information about the child's abuse. In some instances, this may come to fruition, which can lead to treatment refusal or even have iatrogenic effects (e.g., school peer 
finds out about the child's abuse and this leads to unwanted peer interactions). The aforementioned concern that CSA is a topic too private, personal, or stigmatizing to discuss with a therapist may be exacerbated in a group format causing both the victim and the nonoffending caregiver to resist initiating and engaging in treatment (Finkelhor \& Browne, 1985; Theimer \& Hansen, 2018). If a child resists going to therapy for these reasons, but their caregiver insists they go, the feeling of being "forced" to attend can impact engagement and group dynamics independent of the youth's initial rationale for resisting participation. Finally, when participating in group, youth and caregivers may feel disconnected from one another or more stigmatized based on differences in the child's abuse experience (e.g., offender is an adult family member vs. peer, cultural beliefs regarding mental health and trauma) hindering treatment engagement (Brooks \& Hopkins, 2017; Fraynt et al., 2014). However, what some group members perceive as a challenge, others may view as an incentive. Group therapy offers a safe environment for families to discuss their emotions with others who have experienced something similar (van Toledo \& Seymour, 2013). This is beneficial because some may find it difficult to talk openly about sexual abuse with friends and family (Deblinger et al., 2001).

A parent's attendance in therapeutic services is important, as it has been shown to associate with their child's completion of therapy for CSA (McPherson, Scribano, \& Stevens, 2012). When a parent engages in concurrent and integrated services with their child, their own concerns as a parent can be addressed and parents can increase their ability to support their child (Deblinger et al., 2001; McPherson et al., 2012; Stauffer \& Deblinger, 1996).

When parents are able to take the first steps of initiating and participating in group treatment, other barriers can impact treatment adherence. Once in group, parents may have strong negative emotional responses to hearing other families' stories and experiences. If the emotional response is strong enough, further engagement in treatment can be impacted. In addition, parents may perceive differences between their own child's experiences and other group members' children's experiences, including the severity of CSA and the effects of the abuse. Parents who feel their child's abuse was "less severe" compared to others may perceive themselves as different or less worthy of treatment. Caregivers' sense of group cohesion can be 
influenced by these perceptions and parents may be less motivated to attend future sessions.

Similarly, the relationship each caregiver has with their child's offender will likely differ among group members and can cause group cohesion and participation challenges throughout treatment. If strong negative emotions are expressed toward offenders during group sessions, parents may disengage or completely withdraw if they have different emotions toward their child's offender. For example, when a parent's older biological child offends against their younger sibling. While this parent may feel significant anger, they may also hold a range of mixed emotions toward their child's offender, including love, support, and the hope for treatment rather than harsh punishment. Thus, if other group members express extreme anger toward their child's perpetrator (e.g., "I want to hurt them") or hatred toward offenders as whole, parents with complex relationships to the offender may feel ostracized, hindering engagement and reducing the therapeutic environment.

As mentioned previously, high levels of parental self-focus can impact treatment engagement and effectiveness. In addition to impacting individual treatment, this dynamic can affect group treatment as there may be a shift to providing support for that specific parent rather than fulfilling actual treatment goals for supporting the group members' children.

Given that developmentally appropriate sex education is often a part of CSA treatment (Cohen et al., 2017; Hubel et al., 2014), parents' cultural and religious beliefs about sex can impact group dynamics and engagement. Strong beliefs and varying levels of acceptance about sexuality (e.g., related to same-gender relationships, gender identity, masturbation, contraception, sex outside of marriage) may lead to negative interactions among group members who hold differing viewpoints. Parents may refuse for their child to get certain information during session that the other group members are receiving, which can be challenging for therapists to navigate and create a feeling of division among the youth in group. They may also not attend sessions focused on sex education, which reduces attendance and engagement in treatment and may negatively impact group cohesion. 


\subsection{Systemic and societal factors}

Moving toward a broader lens, factors related to the legal system and society can introduce difficulties in treatment initiation, adherence, and effectiveness following CSA. Integrating systemic and societal factors with the barriers related to individual characteristics, perceptions and beliefs, and family and group interaction is critical for understanding the circumstances in which families seek and engage in therapy.

After disclosure of CSA, the legal system may become a significant context for the family. Following one or more forensic interviews, families may frequently meet with detectives and prosecutors to provide additional information, complete confrontation calls or texts with the offender, receive updates on the case, and prepare for court proceedings. While the legal system is intended to provide justice after disclosure of CSA, families in a recent study reported avoiding actions that could involve authorities, such as initiating treatment, because they were intimidated by the authorities' approach, felt excluded from the investigative process, or feared not knowing what would happen next (Collin-Vezina, De La Sablonniere-Griffin, Palmer, \& Milne, 2015). Such concerns can be compounded for families from diverse cultures as language and cultural differences may contribute to miscommunication between the authorities and the family. Additionally, the effects of perceived biases and racism for these families can exacerbate distrust and disengagement with authorities in general (Fontes \& Plummer, 2010; Okamura, Heras, \& Wong-Kernberg, 1995). Thus, families may be apprehensive about initiating and engaging in treatment because of the possibility of authorities needing to be involved if more information regarding CSA is disclosed in therapy.

Likewise, treatment adherence may be impacted by ongoing experiences families have with the legal system. Negative experiences with the legal system (e.g., case was dropped against the offender) can induce painful emotions that evoke unpredictable changes in treatment (Alaggia et al., 2019). For example, a family previously engaged in treatment may have their legal case dropped, resulting in the family withdrawing from treatment due to distrust in the system and hopelessness that the broader system will keep their child safe. Glaser (1991) found that uncertainties regarding verdicts and outcomes 
for the child and the offender can reduce treatment effectiveness due to the impeding presence of the unresolved case. Theoretically, the legal system is devoted to objectivity and evidence; however, prejudice and biases can permeate the justice system and cause unpredictable changes in treatment. For example, Bottoms, Davis, and Epstein (2004) found that mock jurors considered Black and Hispanic children to be more responsible for their CSA compared to White children. Moreover, the defendant was said to be less responsible for sexually abusing a child when the victim was Black compared to White. Such biases lead families to experience prejudices and inequitable legal processes, resulting in distrust of the system which can create a belief that engaging in treatment will also hold undesirable consequences. Therefore, treatment providers need to consider how negative outcomes in legal proceedings can hinder adherence to treatment and client progress (Alaggia et al., 2019).

Within the legal context, custody issues can also serve as a barrier to treatment following CSA. Custody may be especially salient when a child was sexually abused while in one non-offending caregiver's care (e.g., by a mother's boyfriend), which leads the other caregiver to file for full custody of the child (Faller, 1991). Custody issues affect both treatment initiation and adherence. For instance, caregivers may not want to engage in treatment following disclosure for fear of an unfavorable outcome in custody proceedings, including losing custody of the child. During treatment, participants may become so invested in influencing the outcome of custody disputes that they are unwilling or unable to express and process genuine emotions. The child may feel responsible for strained relationships associated with the legal proceedings, impacting their comfort and engagement in treatment.

In terms of societal barriers, stigma the family perceives from others can influence treatment. While one's own feelings of self-blame and guilt were previously discussed, it is important to recognize the influence of broader societal factors on an individual's thoughts and feelings about the abuse (Coffey, Leitenberg, Henning, Turner, \& Bennett, 1996). Particularly, the worse the stigma or shame the family perceives from others, the higher the likelihood that the family will present with more problems related to treatment adherence (Alaggia et al., 2019). Collin-Vezina et al. (2015) found that adult victims of CSA feared that others would blame them, be angry at them, or reject them 
if they knew about the CSA. Moreover, stigma associated with being treated exclusively as a "victim" (Holguin \& Hansen, 2003; Theimer \& Hansen, 2018) and taboos surrounding sex and sexuality may impact engagement in treatment. When working with culturally diverse populations, it is particularly important to understand the source and function of stigma for each distinct culture. While there are individual differences in beliefs and practices within cultures, some cultural beliefs may stigmatize victims and families and, thus, hinder treatment following CSA. For example, in the group treatment context, cultural differences may contribute to certain children feeling disconnected from other group members, particularly if these youth feel that the majority culture group members are less inviting and supportive of the minority culture group members. Moreover, a collectivistic emphasis may create unique barriers to initiation if a greater value is placed on protecting the family as a whole than seeking treatment for an individual (e.g., protecting family against dissolution, threatening community and family integrity, and sharing personal matters with those outside of the community; Fontes \& Plummer, 2010; Futa et al., 2001; Haboush \& Alyan, 2013; Sawrikar \& Katz, 2017).

Differential values of sex among males and females (e.g., sexual scripts where men want and enjoy sex whereas women must be modest and pure) can create additional barriers to seeking treatment following CSA (Fontes \& Plummer, 2010). Sexual scripts make it difficult for male victims to disclose and seek treatment for CSA due to societal views of masculinity that either hinder identification of abuse (e.g., the perpetrator is an older female and therefore seen as a conquest rather than a predator; Fontes \& Plummer, 2010) or promote toxic beliefs about strength and vulnerability (e.g., a male victim may fear the consequences to his masculine image if he admits he was hurt; Alaggia, 2010). Furthermore, Collin-Vezina et al. (2015) found that male victims whose perpetrator was also male feared homophobic responses from others or perceived threats to their masculinity (e.g., boys are tough, boys do not cry) when disclosing CSA.

Additionally, identifying as lesbian, gay, bisexual, transgender, or gender diverse may present additional risks for victimization and barriers to treatment for CSA. In a recent meta-analysis, it was reported that sexual and gender minority (SGM) individuals are 3.8 times more likely to experience CSA compared to their sexual 
non-minority peers (Friedman et al., 2011). Specifically, Alessi, Kahn, and Chatterji (2016) found that SGM children were verbally, physically, and sexually abused by peers and school officials due to their perceived sexual orientation or gender identity. SGM children reported that they were not able to go to their parents due to parental beliefs that they had brought the abuse upon themselves due to their sexual or gender diversity (Alessi et al., 2016). It is evident that gender and sexual identity can serve as a significant risk factor for experiencing CSA and that stigma may be permeating at the familial and societal levels. The societal stigma that sexual and gender identity influences abuse may even decrease supportive families' initiation in treatment. If a victim and their family do engage in treatment, it is possible that they may be fearful and distrusting of the therapist due to past experiences of stigma, which can further impact treatment engagement and effectiveness.

Finally, the field of CSA is multidisciplinary in nature due to the different types of services required to address individual and societal needs (e.g., medical providers, investigators, therapists, advocates, lawyers). While the array of professional expertise is necessary and beneficial, it can also serve as a source of confusion and frustration from the family's perspective. For example, the family may receive contradicting responses from various professionals (Glaser, 1991). Or, the family may become confused about confidentiality practices and question what they are allowed to share with diverse professionals as well as how much the professionals share with each other. Moreover, if one professional disappoints the family (e.g., advocate closes case, medical provider does not find physical evidence), the family may come to distrust or hold negative emotions toward all professionals involved, including therapists. Plummer and Eastin (2007) found that mothers who had no previous interactions with Child Protective Services (CPS) for CSA were surprised by the idleness and insensitivity with which the justice system responded to their child's case and expressed regret in ever contacting the authorities for assistance. This alarming finding suggests that families experience significant frustrations with multisystemic procedures following CSA that may impact their engagement in treatment. 


\section{Recommendations for clinical practice}

\subsection{Prior to treatment initiation}

When working with children, adolescents, and their non-offending family members, therapists should educate themselves on the common barriers to treatment initiation and attempt to make accommodations in their practices that will increase the chances every family has access to therapy. Improving access may include obtaining funding to provide solutions to practical barriers (e.g., financial, transportation, childcare). For example, offering an inclusive environment for other children in the household, such as a waiting room with toys and a skilled supervisor, or treatment programming specific to siblings (Tavkar \& Hansen, 2011) may increase the chances a family is able to begin treatment. Additionally, therapists should provide referrals, as appropriate, to assist with basic needs which may impact treatment initiation. Developing a relationship with community agencies that are part of the multidisciplinary service array for families who experience CSA (e.g., CPS, Child Advocacy Centers, Head Start, Public Schools) can increase community acceptance of interventions. Mental health providers can establish connections within communities to foster trust within diverse populations. With outreach, accurate descriptions of treatment programs as inclusive, supportive, and respectful of family's cultural values may decrease any uncertainties families feel. Clinicians should engage in training to increase their cultural competence (e.g., understanding differences between collectivist and individualistic cultures, cultural beliefs and practices regarding gender roles, stigma associated with victimization). An integrative model of care, in which families can receive mental health, medical, advocacy, and legal services all in one child-friendly building, can reduce the burden placed on families (McPherson et al., 2012; Tavkar \& Hansen, 2011). Further, therapists must be prepared to assess family resources and identify those with whom communication may be needed, such as medical providers, case workers, and those with custody or care rights for the child. Efforts should be made to foster early communications among professionals and all those involved in the case.

Finally, clinicians and researchers should develop interventions to increase treatment accessibility, particularly for underserved 
populations. For example, Community Outreach Program-Esperanza (COPE) was created to "address cultural barriers by offering culturally-modified, evidence-based trauma treatments, led by bilingual/bicultural clinicians, for Hispanic children and families" (Jones et al., 2014, p. 399). COPE services are typically provided in the family's home, church, library, or the child's school to increase access to care (Jones et al., 2014). Telehealth programs can further reduce practical barriers to treatment (Jones et al., 2014; Stewart, Orengo-Aguayo, Cohen, Mannarino, \& de Arellano, 2017).

\subsection{Beginning of treatment}

Once a family decides to initiate mental health services, there are a variety of steps clinicians can take at the beginning of treatment to increase adherence. First, when completing informed consent and assent, therapists should clearly define rules of confidentiality. It is helpful to determine if any family members have questions or concerns about confidentiality or the practical application of those rules. This includes carefully defining what therapists can share with legal authorities and other professionals as well as release of information practices and responses to court orders. During the intake or first session, clinicians should gather information about the child and family that could act as potential risks for disengagement or early dropout. This may include demographic information (i.e., socioeconomic status) as well as perceptions of treatment relevance, any hesitations, and anticipated treatment effectiveness (de Haan et al., 2013). Providers should consider the role of caregivers, siblings, and extended family in treatment approaches and case conceptualization. Therapists should address the family's beliefs, values, protective factors, and identified supports early in treatment and incorporate them as appropriate. It is also important to ask how clients interpret their experiences within the context of their background and tailor interventions accordingly.

Building a strong therapeutic alliance with the client is imperative in trauma-informed mental health services (Yasinski et al., 2018); thus, it may be helpful to devote time early in treatment to build rapport, ensure that therapists understand individual, family, and cultural values of the family, and support the child and parent as they become comfortable talking about sensitive topics. Importantly, clinicians should be aware of their own cultural biases to maintain a 
sensitive therapeutic alliance and to provide culturally competent services. At the beginning of treatment, clinicians can discuss and set expectations for therapy by presenting treatment topics to both the child and caregiver. Therapists should provide the rationale for the importance of treatment topics, particularly sex education and trauma processing, as these may be linked to avoidance (Asmundson et al., 2004). Further, addressing client concerns based on previous therapy experiences can be beneficial early in treatment.

Finally, if one caregiver declines treatment, therapists should process the subsequent emotions for the rest of the family and offer some education on effective family communication. Clinicians can also offer a session between both caregivers to facilitate better communication, as it is possible that a caregiver who initially declines to participate will agree to come for a single information exchange and communication session. Sometimes, very brief psychoeducation and exploration of the personal factors that prevented the person from initially engaging can improve family communication and functioning and may even elicit more engagement. At a minimum, a session with both caregivers can provide useful data for the therapist to consider as they continue to treat the rest of the family.

\subsection{Throughout treatment}

There are a variety of steps clinicians can take throughout treatment to increase engagement and effectiveness. First, therapists should provide children and non-offending family members with psychoeducation about sexual abuse (Hubel et al., 2014), including the definition and general prevalence of sexual abuse and the heterogeneity of outcomes. Additionally, this should include educating parents on the importance of their belief and support following the child's disclosure. Further, mental health providers should address any maladaptive cognitions and perceptions families have during treatment. This may involve discussing expectations the child or parent has following CSA, including addressing some caregivers' concerns that the child is now "damaged" or that the child will never be the same (Kouyoumdjian et al., 2009) and providing information to combat this perception. Moreover, therapists should address parent confusion about how to respond to problematic child behaviors following abuse. Additionally, since selfblame and guilt are common responses to CSA for both 
youth and their non-offending caregivers (Feiring et al., 2002), mental health providers should process these emotions to increase engagement in treatment. Relatedly, if the child was abused in one nonoffending caregiver's household, clinicians should promote effective parenting with both caregivers and address any guilt the child may have due to familial tensions. Similarly, one non-offending caregiver may place blame on the other non-offending parent if the abuse occurred in their home. Therapists should process these emotions, problem-solve with caregivers about how to best support and protect their child in the future, and promote the recognition of the responsibility of the offender. When youth and parents have an ongoing relationship with the offender, therapists must approach the discussion about offenders with sensitivity and address the reality that relationships with offenders can be complicated.

Of importance, providers should be prepared to address different perspectives and beliefs about the abuse that may be influenced by the client's cultural background (Fontes \& Plummer, 2010). For example, children and caregivers from more collectivist cultures may be more concerned about how open discussion of the abuse will continue to affect their family and community than families from more individualistic cultures. Therefore, clinicians need to make sure they help clients process the emotions associated with their distinctive cultural values. As with the beginning of therapy, therapists should continue to be cognizant of any cultural and religious beliefs that may be impacting treatment engagement and contributing to dropout (Fraynt et al., 2014). When providers and clients are from the same culture, therapists need to be cautious that they distinguish their individual understanding of cultural values from those of their clients. When therapists and clients are from different cultures, therapists must take time to understand differing cultural and religious factors. In all, providers should abide by the culturally competent practice guidelines, recognizing that each cultural group is not homogenous and not imposing their own cultural stereotypes on clients. Treatment should be sensitive to the increased safety risks faced by SGM children, while including psychoeducation for their families about victims not being at fault for CSA because of their gender or sexual identity. Further, clinicians should help families who disapprove of their child's gender identity or sexual orientation to be supportive of their children through treatment. 
It is important to consider CSA within the legal context; therefore, as appropriate, mental health providers can provide accurate information or referrals regarding legal system proceedings in CSA cases. This may include informing families on statistics of CSA cases ending in a conviction and processing the case and any frustrations with the legal system as the case proceeds.

Education about age-appropriate sexual development and behavior increases positive outcomes (Cohen et al., 2017; Hubel et al., 2014), thus, therapists need to ensure sex education is delivered in a way that parents find it applicable to their family situation. Clinicians should identify any concerns parents have about sex education and process the emotions associated with those concerns. Therapist should also be open with parents about the fact-based information they will share with their child.

As therapy continues, clinicians should promote adaptability in treatment length and structure to address changing needs. Particularly, changes in family environments and ongoing parental stress can have impacts for both non-offending family members and CSA survivors. Finally, if caregivers repeatedly focus on their own CSA history, therapists should provide referrals and encourage individual therapy that can specifically attend to a parent's own sexual abuse story (van Toledo \& Seymour, 2013). Clinicians should explain the advantages of seeking mental health services to process emotions related to caregivers' own experiences (van Toledo \& Seymour, 2013).

\subsection{Group therapy - beginning of treatment}

While the above recommendations apply to group treatment contexts, additional and special recommendations exist for therapists who provide group therapy for children, adolescents, and non-offending family members impacted by CSA. First, a parent's engagement in parallel group therapy can be an important protective factor for families; thus, providers should encourage parent involvement in group services, as appropriate. To help group members feel more comfortable about confidentiality, therapists should spend time building rapport and reviewing the specifics of confidentiality (e.g., what group members say is private and should not be discussed outside of group) and why confidentiality is important. In addition, during the first session 
of group (or when members join), clinicians can have a conversation about what should happen if participants see each other outside of group or what should happen if members know each other. If prior relationships exist among members, therapists and group participants should have a private conversation to make sure participation is appropriate and that everyone feels comfortable going forward. Therapists should also be aware of any cultural and religious beliefs that are contributing to concerns of confidentiality (Brooks \& Hopkins, 2017; Fraynt et al., 2014). Gaining group participants' trust contributes to their ability to learn skills throughout treatment, fostering better outcomes.

Cultural influences may also impact the level of comfort an individual has with sharing during group therapy (Brooks \& Hopkins, 2017; Fraynt et al., 2014). Thus, therapists should contemplate cultural considerations when developing group treatments, particularly for CSA (Atdjian \& Vega, 2005; Bernard, 1999). Also, clinicians should promote an atmosphere of respect that allows group members to share as much or as little information as they feel comfortable disclosing in the moment. It may be necessary to remind group members that there are many different perspectives, and to be respectful of different opinions. Particularly, parents may have strong beliefs about sex education, which may impact group dynamics and engagement. As described before, therapists should describe the importance of sex education for a child after experiencing sexual abuse (Cohen et al., 2017; Hubel et al., 2014) and discuss with parents any concerns they may have privately and ultimately respect parental decisions.

\subsection{Group therapy - throughout treatment}

Throughout treatment, several factors can increase adherence and effectiveness for group members. Typically, discussions about feelings related to the sexual abuse occur in group treatment. Talking about sexual abuse can be intimidating for many victims, and the group context may create additional reluctance for some. When therapists reflect group members' emotions regarding discussing CSA, it validates those emotions and encourages discussion. When clinicians point out similarities among members, it increases cohesiveness and is especially helpful for group members who feel disconnected from one another due to differences in their abuse histories. For example, although 
their actual abuse experiences may differ, emotions about it may be much more alike. Conversely, when group members are reporting different emotions about the abuse, therapists should normalize the contrasting responses by stating that there is no one "correct" way to feel about what happened and that people often have unique emotional experiences in response to CSA.

Similarly, therapists should be mindful when group members have different emotions about the offender. If these different and intense emotions start to cause group cohesion issues and contribute to disengagement for some group members, clinicians should check in with the group members after session in private. This is an opportunity for the therapist to gain a better understanding of how a particular participant feels about therapy in a group setting. During group, therapists should make a statement about respecting each other and recognizing that everyone may have different emotions about the offender. It is important to normalize emotional variation among group members' reactions to their offenders.

Finally, if parents have their own abuse history, their attention may be focused away from the goal of group. Clinicians should speak to individuals with the abuse history in private about services available to help them process their own abuse and free them to focus on their child's experience during the CSA treatment group. If they do not have an individual provider, a referral should be given (van Toledo \& Seymour, 2013). Therapists should promote individual problem-solving for concrete steps to focusing on their child and the needs of the other families in the group.

\subsection{Therapist factors}

A variety of therapist-related factors were briefly described above as they relate to clinicians who primarily work with children and families who experience CSA. However, it is important to note that some therapists who do not specialize in CSA will end up working with clients who have histories of sexual abuse, and there are special recommendations for these circumstances. For example, a provider may be seeing a child for a separate issue (e.g., depression, anxiety, behavioral problems) when the client discloses sexual abuse during the course of treatment. As always, all providers must be aware of reporting issues surrounding CSA and discuss any concerns regarding this with 
the client and their family. Rules of confidentiality should be part of ongoing conversations covering consent in treatment. Beyond reporting issues, however, a clinician must decide which intervention will fit the child and family's needs best. Specifically, if the therapist does not have the clinical expertise and training to provide traumainformed, evidenced- based therapy or if the clinician does not feel they can provide the appropriate care for other reasons, the therapist must provide a referral. Effective referrals require the clinicians to consider how potential service providers ensure that the three pillars of evidence-based practice are integrated (i.e., best available research, clinical expertise, and client characteristics, culture, and preferences; Sackett et al., 1996).

Further, in all circumstances, therapists should be aware of their own characteristics, beliefs, and perceptions, as these factors can impact treatment adherence and effectiveness. Broadly, for clinicians who specialize in CSA or child trauma as well as clinicians who generally work with youth and families, therapist training and sensitivity in the area of CSA (e.g., training to play a supportive role, education regarding CSA-specific issues, understanding treatment barriers) is helpful to ensure all clients receive appropriate services.

\section{Directions for future research}

A dearth of research exists on the barriers to treatment for children, adolescents, and families who experience CSA (McPherson et al., 2012). Future research should focus on identifying and reducing barriers to treatment, informing clinical practice on ways to increase treatment acceptability and effectiveness, and the impact of treatment on shortand long-term outcomes of CSA. Understanding child and caregiver individual characteristics is important for differentiating the source and impact of the range of barriers identified in this paper. For example, understanding how moderating factors (e.g., client-therapist relationship, client perceptions of therapist sensitivity; Yasinski et al., 2018) may influence the association between individual client characteristics and treatment adherence is likely to improve responsiveness to treatment. Similarly, further research could be completed on how these possible moderating factors impact client avoidance, given the important role of avoidance as a presenting symptom 
related to sexual abuse for trauma-focused treatment engagement (Yasinski et al., 2018). More research is also needed on how ongoing youth-offender relationships as well as caregiver history of CSA influence treatment adherence and effectiveness (e.g., how does having a continued relationship with a peer offender impact treatment effectiveness?). Importantly, further research examining deconstructed and reorganized implementations of trauma-focused treatment following CSA would be helpful to inform clinical practice (e.g., does moving sex education earlier or later in treatment increase retention and engagement?).

Research related to child and caregiver perceptions and beliefs is also needed. Specifically, while some research has examined the predictors of parents' negative expectations for their child following CSA (Kouyoumdjian et al., 2009; Morrissey-Kane \& Prinz, 1999), more studies are needed on the long-term effects of parents' expectations on child functioning and how negative expectations can be challenged in treatment (e.g., what are effective strategies in reducing negative parental expectations following CSA?). Further, more literature is warranted surrounding the risk and protective factors for developing maladaptive perceptions and beliefs as well as how CSA-related perceptions vary by culture (Fontes \& Plummer, 2010).

Since CSA impacts the entire family (Cyr et al., 2016), research on interventions that account for family interaction-related barriers to treatment could help increase engagement (Hubel et al., 2014). A continued and updated understanding on the changing needs of families in today's socio-environmental context is important for providing the best services to all family members. Similarly, studies examining how culture influences family dynamics and parenting following CSA and how these relate to treatment outcomes would be beneficial (e.g., do collectivist and individualist cultures benefit from different methods of treatment for CSA?).

While the empirical literature supports the effectiveness of group treatments for CSA (Deblinger et al., 2001; Hubel et al., 2014; van Toledo \& Seymour, 2013), more research is needed to better understand barriers to group therapy. For example, studies should examine how client-therapist relationship and alliance among group members relates to treatment engagement and effectiveness. Further, beyond clinical experience, little research exists on how differences in abuse experiences among group participants impacts engagement (e.g., 
does having differing CSA experiences among group members provide more opportunities for insight, group-processing, and support in CSA treatment?).

Recognizing the importance of the systemic and societal context is critical when completing research on CSA treatments. Similarly, while cultural influences on disclosure have been examined and reviewed (Alaggia et al., 2019; Brazelton, 2015; Fontes \& Plummer, 2010), further investigation of the various cultural influences on CSA treatment initiation, adherence and dropout, and effectiveness are needed. Specifically, the development, dissemination, and implementation of culturally adapted treatment for survivors of CSA and their non-offending caregivers is critical for providing supportive services to all those in need. Further research examining how gender identity, gender norms, and sexual orientation as well as how the gender of the offender influences a client's willingness to express their emotions about the abuse is needed. Of note, researchers should be aware of the influences of culture on treatment outcome measures. Some groups may perform differently than normed samples, and further exploration of these potential differences is warranted.

Finally, while telehealth programs are promising and can reduce some of the practical barriers to treatment (Jones et al., 2014), further research on the utilization of telehealth for families impacted by CSA and finding solutions to the challenges of telehealth are needed (Stewart et al., 2017).

\section{Considerations for policy change}

While clinical work and research inform each other in providing qual-

ity care, changes at the policy level can further promote standards of care and address public misperceptions when working with children who have experienced CSA and their families.

\subsection{Train professionals and authorities on CSA}

To decrease barriers to treatment, it is necessary that policy development focuses on increasing training for mental health professionals, authorities, and all those involved in the multidisciplinary team to promote knowledge, sensitivity, and cultural awareness when working 
with families impacted by CSA. As noted, many families feel disregarded, confused, and frustrated when working with authorities after disclosure (Collin-Vezina et al., 2015), which can impact treatment initiation and engagement. Thus, it is important that legal professionals receive training on sensitive communication with families following CSA to promote trust and understanding between the family and the authorities. Further, policy can inform training and practice guidelines for mental health clinicians. For example, children and caregivers may be avoidant of therapy for CSA and research demonstrates that in-session avoidance predicts child outcomes (Yasinski et al., 2016) and dropout (Yasinski et al., 2018). Therefore, it is pertinent that mental health professionals are competent in identifying and addressing avoidance in session in a trauma-informed manner. Finally, it is necessary that all professionals develop a stronger sense of cultural competency when working with diverse populations affected by CSA. Specifically, previous research has found that many minority status individuals (e.g., ethnic, sexual, gender) face additional barriers due to cultural or language differences (Fontes \& Plummer, 2010; Okamura et al., 1995) and biases regarding responsibility in abuse (Bottoms et al., 2004). It is evident that additional training focused on cultural sensitivity should be promoted within the legal system as well as in policy development to diminish the negative experiences some children and families face after disclosure of CSA. Overall, efforts at the policy level must be taken to ensure professionals and authorities are reducing treatment barriers, best supporting families, and increasing positive outcomes.

\subsection{Increase public knowledge to change public perceptions}

Policy makers should also consider how to best address inaccurate public perceptions of CSA and educate the general public with accurate information regarding prevalence, characteristics of perpetrators, and victim outcomes, which could reduce the societal stigma surrounding seeking treatment for CSA. For example, stigma contributes significantly to a family's decision to initiate and engage in treatment. Research has found that the worst stigma a family experiences, the higher likelihood they will present with additional problems related to treatment adherence (Alaggia et al., 2019). Further, while research findings within the field of CSA have debunked common public 
misconceptions (e.g., CSA survivors are "damaged goods," perpetrators of CSA are most often strangers), some myths continue to persist today. Due to this, it appears that research findings negating misconceptions about CSA have not been disseminated well to the general public. Moreover, the stigma of reporting and discussing CSA can be particularly evident in minority communities (Futa et al., 2001). Therefore, special efforts to educate and provide resources to underserved communities are pertinent to increasing rates of disclosure and increasing initiation and adherence to treatment.

Furthermore, public awareness of the importance of sex education following CSA is necessary to increase understanding of developmentally appropriate sexual knowledge and behaviors. Specifically, when caregivers are not aware of age-appropriate sexual development, they may not recognize if their child is engaging in inappropriate sexual behavior-which may further exacerbate the child's sexualized behavior problems. Alternatively, a caregiver may believe that a child's developmentally appropriate behavior is a consequence of the sexual abuse. Due to this, it is necessary that policy enacts to promote developmentally appropriate sex education to youth and their caregivers, especially after the experience of sexual abuse.

Finally, providing public awareness on evidence-based trauma-informed and trauma-focused therapy is necessary in promoting positive outcomes in children and their families. Specifically, the application of evidence-based treatments work to improve caregivers' expectations of treatment and emphasize the importance of involving non-offending family members in treatment. Given that insurance companies require a mental health diagnosis to cover treatment, therapy utilization may be difficult for non-offending family members and demonstrates a need for policy reform in mental health care. Overall, the need for public knowledge regarding the effectiveness of evidence-based treatment is important in promoting positive outcomes for both the victim and their non-offending family members after disclosure.

\section{Conclusion}

There is clear consensus that survivors of CSA deserve accessible and effective mental health treatment, and there is increasing evidence that addressing barriers to treatment is necessary. A critical 
examination of the barriers to successful treatment reveals that, in addition to the practical obstacles common to mental health service utilization, such as transportation and child care, child sexual abuse victims face CSA-specific barriers that are complex and interrelated. Understanding the distinct barriers to CSA treatment can help clinicians, researchers, and policy makers improve efforts to support child victims and their non-offending caregivers in overcoming them. This paper detailed impediments to CSA treatment initiation, adherence, and effectiveness by exploring associated child and caregiver characteristics, social and individual beliefs and perceptions, family relationships, dynamics present in group treatment, and how broader systemic and societal factors may compound barriers. This exploration led to recommendations for therapeutic intervention strategies designed to address the barriers directly and empower therapy participants to find ways to benefit from treatment in spite of difficulties. Exploration of CSA-specific treatment barriers pointed to new and ongoing research directions and suggested policy considerations, including addressing multidisciplinary training needs and improving public awareness to correct common misconceptions and biases about childhood sexual abuse.

Declaration of competing interest - The authors declare there are no competing interests.

Acknowledgements - The authors would like to thank all of the past Project SAFE therapists who provided their time to serving families and contributed to our understanding of and addressing treatment barriers. In particular, we would like to thank Jeremy Holm for his valuable work on this topic.

\section{References}

Alaggia, R. (2010). An ecological analysis of child sexual abuse disclosure: Considerations for child and adolescent mental health. Journal of the Canadian Academy of Child and Adolescent Psychiatry, 19, 32-39.

Alaggia, R., Collin-Vezina, D., \& Lateef, R. (2019). Facilitators and barriers to child sexual abuse (CSA) disclosures: A research update (2000-2016). Trauma, Violence, \& Abuse, 20, 260-283.

Alessi, E. J., Kahn, S., \& Chatterji, S. (2016). "The darkest times of my life": Recollections of child abuse among forced migrants persecuted because of their sexual orientation and gender identity. Child Abuse \& Neglect, 51, 93-105. 
Asmundson, G. J., Stapleton, J. A., \& Taylor, S. (2004). Are avoidance and numbing distinct PTSD symptom clusters? Journal of Traumatic Stress, 17, 467-475.

Atdjian, S., \& Vega, W. A. (2005). Disparities in mental health treatment in US racial and ethnic minority groups: Implications for psychiatrists. Psychiatric Services, $56,1600-1602$.

Banyard, V. L., Rozelle, D., \& Englund, D. W. (2001). Parenting the traumatized child: Attending to the needs of nonoffending caregivers of traumatized children. Psychotherapy: Theory, Research, Practice, Training, 38, 74-87.

Bernard, C. (1999). Child sexual abuse and the black disabled child. Disability and Society, 14, 325-339.

Bolen, R. M., \& Lamb, J. L. (2004). Ambivalence of nonoffending guardians after child sexual abuse disclosure. Journal of Interpersonal Violence, 19, 185-211.

Bottoms, B. L., Davis, S. L., \& Epstein, M. A. (2004). Effects of victim and defendant race on Jurors' decisions in child sexual abuse cases 1. Journal of Applied Social Psychology, 34, 1-33.

Brazelton, J. F. (2015). The secret storm: Exploring the disclosure process of African American women survivors of child sexual abuse across the life course. Traumatology, 21, 181.

Bronfenbrenner, U., \& Morris, P. A. (2006). The bioecological model of human development. In R. M. Lerner, \& W. Damon (Eds.). Handbook of child psychology: Theoretical models of human development (pp. 793-828). Hoboken, NJ: John Wiley \& Sons.

Brooks, R. T., \& Hopkins, R. (2017). Cultural mistrust and health care utilization: The effects of a culturally responsive cognitive intervention. Journal of Black Studies, 48, 816-834.

Coffey, P., Leitenberg, H., Henning, K., Turner, T., \& Bennett, R. T. (1996). The relation between methods of coping during adulthood with a history of childhood sexual abuse and current psychological adjustment. Journal of Consulting and Clinical Psychology, 64, 1090-1093.

Cohen, J. A., \& Mannarino, A. P. (1996). A treatment outcome study for sexually abused preschool children: Initial findings. Journal of the American Academy of Child \& Adolescent Psychiatry, 35, 42-50.

Cohen, J. A., \& Mannarino, A. P. (1998). Interventions for sexually abused children: Initial treatment outcome findings. Child Maltreatment, 3, 17-26.

Cohen, J. A., Mannarino, A. P., \& Deblinger, E. (2017). Treating trauma and traumatic grief in children and adolescents (2nd ed.). New York, NY: Guilford Press.

Collin-Vezina, D., De La Sablonniere-Griffin, M., Palmer, A. M., \& Milne, L. (2015). A preliminary mapping of individual, relational, and social factors that impede disclosure of childhood sexual abuse. Child Abuse \& Neglect, 43, 123-134.

Craun, S. W., \& Theriot, M. T. (2009). Misperceptions of sex offender perpetration: Considering the impact of sex offender registration. Journal of Interpersonal Violence, 24, 2057-2072.

Craven, S., Brown, S., \& Gilchrist, E. (2007). Current responses to sexual grooming: Implication for prevention. The Howard Journal of Criminal Justice, 46, 6o-71. 
Cyr, M., Frappier, J. Y., Hebert, M., Tourigny, M., McDuff, P., \& Turcotte, M.E. (2016). Psychological and physical health of nonoffending parents after disclosure of sexual abuse of their child. Journal of Child Sexual Abuse, 25, 757-776.

de Haan, A. M., Boon, A. E., de Jong, J. T., Hoeve, M., \& Vermeiren, R. R. (2013). A metaanalytic review on treatment dropout in child and adolescent outpatient mental health care. Clinical Psychology Review, 33, 698-711.

Deblinger, E., Stauffer, L. B., \& Steer, R. A. (2001). Comparative efficacies of supportive and cognitive behavioral group therapies for young children who have been sexually abused and their nonoffending mothers. Child Maltreatment, 6 , 332-343.

Domhardt, M., Munzer, A., Fegert, J. M., \& Goldbeck, L. (2015). Resilience in survivors of child sexual abuse: A systematic review of the literature. Trauma, Violence, \& Abuse, 16, 476-493.

Elliott, A. N., \& Carnes, C. N. (2001). Reactions of nonoffending parents to the sexual abuse of their child: A review of the literature. Child Maltreatment, 6, 314-331.

Faller, K. C. (1991). Possible explanations for child sexual abuse allegations in divorce. American Journal of Orthopsychiatry, 61, 86-91.

Feiring, C., \& Cleland, C. (2007). Childhood sexual abuse and abuse-specific attributions of blame over 6 years following discovery. Child Abuse \& Neglect, 31, 1169-1186.

Feiring, C., Taska, L., \& Chen, K. (2002). Trying to understand why horrible things happen: Attribution, shame, and symptom development following sexual abuse. Child Maltreatment, 7, 25-39.

Finkelhor, D., \& Browne, A. (1985). The traumatic impact of child sexual abuse: A conceptualization. American Journal of Orthopsychiatry, 55, 530-541.

Fontes, L. A., \& Plummer, C. (2010). Cultural issues in disclosures of child sexual abuse. Journal of Child Sexual Abuse, 19, 491-518.

Fraynt, R., Ross, L., Baker, B. L., Rystad, I., Lee, J., \& Briggs, E. C. (2014). Predictors of treatment engagement in ethnically diverse, urban children receiving treatment for trauma exposure. Journal of Traumatic Stress, 27, 66-73.

Friedman, M. S., Marshal, M. P., Guadamuz, T. E., Wei, C., Wong, C. F., Saewyc, E. M., \& Stall, R. (2011). A meta-analysis of disparities in childhood sexual abuse, parental physical abuse, and peer victimization among sexual minority and sexual nonminority individuals. American Journal of Public Health, 101, 1481-1494.

Frothingham, T. E., Hobbs, C. J., Wynne, J. M., Yee, L., Goyal, A., \& Wadsworth, D. J. (2000). Follow up study eight years after diagnosis of sexual abuse. Archives of Disease in Childhood, 83, 132-134.

Futa, K. T., Hsu, E., \& Hansen, D. J. (2001). Child sexual abuse in Asian American families: An examination of cultural factors that influence prevalence, identification, and treatment. Clinical Psychology: Science and Practice, 8, 189-209.

Glaser, D. (1991). Treatment issues in child sexual abuse. The British Journal of Psychiatry, 159, 769-782. 
Gopalan, G., Goldstein, L., Klingenstein, K., Sicher, C., Blake, C., \& McKay, M. M. (2010). Engaging families into child mental health treatment: Updates and special considerations. Journal of the Canadian Academy of Child and Adolescent Psychiatry, 19, 182-196.

Gulliver, A., Griffiths, K. M., \& Christensen, H. (2010). Perceived barriers and facilitators to mental health help-seeking in young people: A systematic review. BMC Psychiatry, 10, 113.

Haboush, K. L., \& Alyan, H. (2013). "Who can you tell?” Features of Arab culture that influence conceptualization and treatment of childhood sexual abuse. Journal of Child Sexual Abuse, 22, 499-518.

Haugaard, J. J. (2000). The challenge of defining child sexual abuse. American Psychologist, 55, 1036.

Hill, A. (2001). 'No-one else could understand': Women's experiences of a support group run by and for mothers of sexually abused children. British Journal of Social Work, 31, 385-397.

Holguin, G., \& Hansen, D. J. (2003). The "sexually abused child": Potential mechanisms of adverse influences of such a label. Aggression and Violent Behavior, 8, $645^{-670 .}$

Hubel, G. S., Campbell, C., West, T., Friedenberg, S., Schreier, A., Flood, M. F., \& Hansen, D. J. (2014). Child advocacy center based group treatment for child sexual abuse. Journal of Child Sexual Abuse, 23, 304-325.

Hunter, J. A., Jr., Goodwin, D. W., \& Wilson, R. J. (1993). Attributions of blame in child sexual abuse victims: An analysis of age and gender influences. Journal of Child Sexual Abuse, 1, 75-89.

Jones, A. M., Shealy, K. M., Reid-Quinones, K., Moreland, A. D., Davidson, T. M., Lopez, C. M., ... de Arellano, M. A. (2014). Guidelines for establishing a telemental health program to provide evidence-based therapy for trauma-exposed children and families. Psychological Services, 11, 398.

Kazdin, A. E., Holland, L., \& Crowley, M. (1997). Family experience of barriers to treatment and premature termination from child therapy. Journal of Consulting and Clinical Psychology, 65, 453.

Kendall-Tackett, K. A., Williams, L. M., \& Finkelhor, D. (1993). Impact of sexual abuse on children: A review and synthesis of recent empirical studies. Psychological Bulletin, 113, 164.

Kerkorian, D., Bannon, W. M., Jr., \& McKay, M. (2006). Seeking help a second time: Parents'/caregivers' characterizations of previous experiences with mental health services for their children and perceptions of barriers to future use. American Journal of Orthopsychiatry, 76, 161-166.

Klonsky, E. D., \& Moyer, A. (2008). Childhood sexual abuse and non-suicidal selfinjury: Meta-analysis. The British Journal of Psychiatry, 192, 166-170.

Kouyoumdjian, H., Perry, A. R., \& Hansen, D. J. (2009). Nonoffending parent expectations of sexually abused children: Predictive factors and influence on children's recovery. Journal of Child Sexual Abuse, 18, 40-6o.

London, K., Bruck, M., Ceci, S. J., \& Shuman, D. W. (2005). Disclosure of child sexual abuse: What does the research tell us about the ways that children tell? Psychology, Public Policy, and Law, 11, 194. 
Malloy, L. C., Mugno, A. P., Rivard, J. R., Lyon, T. D., \& Quas, J. A. (2016). Familial influences on recantation in substantiated child sexual abuse cases. Child Maltreatment, 21, 256-261.

Maniglio, R. (2009). The impact of child sexual abuse on health: A systematic review of reviews. Clinical Psychology Review, 29, 647-657.

McPherson, P., Scribano, P., \& Stevens, J. (2012). Barriers to successful treatment completion in child sexual abuse survivors. Journal of Interpersonal Violence, 27, 23-39.

Miller, R., \& Dwyer, J. (1997). Reclaiming the mother-daughter relationship after sexual abuse. Australian and New Zealand Journal of Family Therapy, 18, 194-202.

Mojtabai, R., Olfson, M., Sampson, N. A., Jin, R., Druss, B., Wang, P. S., ... Kessler, R. C. (2011). Barriers to mental health treatment: Results from the National Comorbidity Survey Replication. Psychological Medicine, 41, 1751-1761.

Morrissey-Kane, E., \& Prinz, R. J. (1999). Engagement in child and adolescent treatment: The role of parental cognitions and attributions. Clinical Child and Family Psychology Review, 2, 183-198.

Okamura, A., Heras, P., \& Wong-Kernberg, L. (1995). Asians, Pacific Island, and Filipino Americans and sexual child abuse. In L. Fontes (Ed.). Sexual abuse in nine north American cultures (pp. 67-96). Thousand Oaks, CA: Sage.

Ormhaug, S. M., \& Jensen, T. K. (2018). Investigating treatment characteristics and first-session relationship variables as predictors of dropout in the treatment of traumatized youth. Psychotherapy Research, 28, 235-249.

Paolucci, E. O., Genuis, M. L., \& Violato, C. (2001). A meta-analysis of the published research on the effects of child sexual abuse. The Journal of Psychology, 135, 17-36.

Plummer, C. A., \& Eastin, J. A. (2007). System intervention problems in child sexual abuse investigations: The mothers' perspectives. Journal of Interpersonal Violence, 22, 775-787.

Roesler, T. A., \& McKenzie, N. (1994). Effects of childhood trauma on psychological functioning in adults sexually abused as children. Journal of Nervous and Mental Disease, 182, 145-150.

Runtz, M. G., \& Schallow, J. R. (1997). Social support and coping strategies as mediators of adult adjustment following childhood maltreatment. Child Abuse \& Neglect, 21, 211-226.

Runyon, M. K., Spandorfer, E. D., \& Schroeder, C. M. (2014). Cognitions and distress in caregivers after their child's sexual abuse disclosure. Journal of Child Sexual Abuse, 23, 146-159.

Sackett, D. L., Rosenberg, W. M., Gray, J. A., Haynes, R. B., \& Richardson, W. S. (1996). Evidence based medicine: What it is and what it isn't. BMJ (Clinical Research Ed.), 312, 71-72.

Sawrikar, P., \& Katz, I. (2017). The treatment needs of victims/survivors of child sexual abuse (CSA) from ethnic minority communities: A literature review and suggestions for practice. Children and Youth Services Review, 79, 166-179. 
Sawyer, G. K., \& Hansen, D. J. (2014). Heterogeneous symptom patterns of sexually abused youth in treatment: Understanding the complexity of the problem. Journal of Child Sexual Abuse, 23, 900-917.

Spataro, J., Mullen, P. E., Burgess, P. M., Wells, D. L., \& Moss, S. A. (2004). Impact of child sexual abuse on mental health: Prospective study in males and females. The British Journal of Psychiatry, 184, 416-421.

Stauffer, L. B., \& Deblinger, E. (1996). Cognitive behavioral groups for nonoffending mothers and their young sexually abused children: A preliminary treatment outcome study. Child Maltreatment, 1, 65-76.

Stewart, R. W., Orengo-Aguayo, R. E., Cohen, J. A., Mannarino, A. P., \& de Arellano, M. A. (2017). A pilot study of trauma-focused cognitive-behavioral therapy delivered via telehealth technology. Child Maltreatment, 22, 324-333.

Tavkar, P., \& Hansen, D. J. (2011). Interventions for families victimized by child sexual abuse: Clinical issues and approaches for child advocacy center-based services. Aggression and Violent Behavior, 16, 188-199.

Theimer, K., \& Hansen, D. J. (2018). Child sexual abuse: Stigmatization of victims and suggestions for clinicians. The Behavior Therapist, 41, 213-219.

Trask, E. V., Walsh, K., \& DiLillo, D. (2011). Treatment effects for common outcomes of child sexual abuse: A current meta-analysis. Aggression and Violent Behavior, 16, 6-19.

Ullman, S. E. (2002). Social reactions to child sexual abuse disclosures: A critical review. Journal of Child Sexual Abuse, 12, 89-121. van Toledo, A., \& Seymour, F. (2013). Interventions for caregivers of children who disclose sexual abuse: A review. Clinical Psychology Review, 33, 772-781.

Wolfe, D. A., \& Jaffe, P. (1991). Child abuse and family violence as determinants of child psychopathology. Canadian Journal of Behavioural Science, 23, 282.

World Health Organization. (1999). Report of the consultation on child abuse prevention. Geneva, Switzerland: World Health Organization.

Yancey, C. T., \& Hansen, D. J. (2010). Relationship of personal, familial, and abuses-specific factors with outcome following childhood sexual abuse. Aggression and Violent Behavior, 15, 410-421.

Yasinski, C., Hayes, A. M., Alpert, E., McCauley, T., Ready, C. B., Webb, C., \& Deblinger, E. (2018). Treatment processes and demographic variables as predictors of dropout from trauma-focused cognitive behavioral therapy (TF-CBT) for youth. Behaviour Research and Therapy, 107, 10-18.

Yasinski, C., Hayes, A. M., Ready, C. B., Cummings, J. A., Berman, I. S., McCauley, T., ... Deblinger, E. (2016). In-session caregiver behavior predicts symptom change in youth receiving trauma-focused cognitive behavioral therapy (TFCBT). Journal of Consulting and Clinical Psychology, 84, 1066.

Zinzow, H., Seth, P., Jackson, J., Niehaus, A., \& Fitzgerald, M. (2010). Abuse and parental characteristics, attributions of blame, and psychological adjustment in adult survivors of child sexual abuse. Journal of Child Sexual Abuse, 19, 79-98. 